\title{
A Dual X-Ray Absorptiometry Validated Geometric Model for the Calculation of Body Segment Inertial Parameters of Young Females
}

\author{
Samantha L. Winter, ${ }^{1}$ Sarah M. Forrest, ${ }^{2}$ Joanne Wallace, ${ }^{2}$ and John H. Challis ${ }^{3}$ \\ ${ }^{1}$ University of Kent; ${ }^{2}$ Aberystwyth University; ${ }^{3}$ The Pennsylvania State University
}

\begin{abstract}
The purpose of this study was to validate a new geometric solids model, developed to address the lack of female-specific models for body segment inertial parameter estimation. A second aim was to determine the effect of reducing the number of geometric solids used to model the limb segments on model accuracy. The full model comprised 56 geometric solids, the reduced model comprised 31, and the basic model comprised 16. Predicted whole-body inertial parameters were compared with direct measurements (reaction board, scales), and predicted segmental parameters with those estimated from whole-body dual x-ray absorptiometry scans for 28 females. The percentage root mean square error (\%RMSE) for whole-body volume was $<2.5 \%$ for all models and $1.9 \%$ for the full model. The \%RMSE for whole-body center of mass location was $<3.2 \%$ for all models. The $\%$ RMSE whole-body mass was $<3.3 \%$ for the full model. The RMSE for segment masses was $<0.5 \mathrm{~kg}(<0.5 \%)$ for all segments; Bland-Altman analysis showed the full and reduced models could adequately model thigh, forearm, foot, and hand segments, but the full model was required for the trunk segment. The proposed model was able to accurately predict body segment inertial parameters for females; more geometric solids are required to more accurately model the trunk.
\end{abstract}

Keywords: joint moment, center of mass, segment mass, moment of inertia

In order to study, analyze, or optimize human movement, the mass, the center of mass location, and the body segment moments of inertia must be known. These body segment inertial parameters (BSIPs) affect the accuracy when calculating the resultant joint moments during activities which involve high accelerations, such as throwing or kicking. ${ }^{1,2}$ Even during the swing-phase of gait, significant differences in resultant joint moments have been calculated as a result of using different methods of estimating the BSIPs of the thigh segment. ${ }^{1}$ Geometric models are a cost-effective way of estimating subject-specific BSIPs, however, a key problem is that no female-specific geometric models for estimating BSIPs have been validated, despite significant differences in the shapes of segments between males and females. ${ }^{3}$

There are several methods of estimating BSIPs. Scanning techniques such as dual x-ray absorptiometry (DXA) have been used to estimate BSIPs., ${ }^{4,5}$ DXA has also been shown to reliably predict subject whole-body mass, ${ }^{6}$ and segmentation of the scan can be customized in order to determine the mass of individual body segments. However, DXA does have some drawbacks, including its cost, general availability, and radiation exposure.

(C) 2018 The Authors. Published by Human Kinetics, Inc. This is an Open Access article distributed under the terms of the Creative Commons Attribution License CC BY 4.0, which permits unrestricted noncommercial and commercial use, distribution, and reproduction in any medium, provided the original work is properly cited, the new use includes a link to the license, and any changes are indicated. See https:// creativecommons.org/licenses/by/4.0/. This license does not cover any third-party material which may appear with permission in the article.

Winter is with the School of Sport and Exercise Sciences, University of Kent, Kent, United Kingdom. Forrest and Wallace are with the Department of Sport and Exercise Science, Aberystwyth University, Ceredigion, United Kingdom. Challis is with the Department of Kinesiology, The Pennsylvania State University, State College, PA. Address author correspondence to Samantha L. Winter at S.L.Winter@kent.ac.uk.
Geometric modeling techniques represent the shapes of the body segments as geometric solids, the dimensions of which (eg, segment length and perimeter) are determined by subjectspecific anthropometric measurements. ${ }^{7-9}$ Mean errors for wholebody mass estimations using geometric models have been as low as $0.3 \%{ }^{7}$ and $1 \% .^{9}$

In contrast with DXA-derived BSIPs, methods based on anthropometric measurements are inexpensive as they require a small amount of readily-available equipment. These measurements can then be used in conjunction with geometric models or regression equations to estimate subject-specific BSIPs. For this reason, although DXA can be used to determine subject-specific BSIPs, it has more practical utility as a validating tool for geometric models, $, 5,10,11$ or to provide segment density values. DXA-validated geometric models have produced accurate BSIP estimations of individual segments such as the shank. ${ }^{10}$

Some studies have assessed the validity of geometric models for some body segments, or for small numbers of females, ${ }^{7,11,12}$ however, there have been no geometric models for biomechanical analysis developed primarily for females and then validated for all body segments for a large participant pool. Bellemare et $\mathrm{al}^{3}$ noted significant differences in the shape of the thoracic region of the trunk between males and females; if used for both sexes, a geometric model should be able to account for these sex-specific differences. The appropriate geometric solid model of the trunk must be detailed enough to take into account the differences in shape of the trunk between the sexes, ${ }^{3}$ and the nature of the crosssection of the trunk. ${ }^{13}$

The purpose of this study was to validate a geometric solid model specifically defined for female subjects by comparing model predicted data with direct whole-body measurements and DXAderived segmental mass data. The model was similar to that of Yeadon ${ }^{9}$ but adapted such that an increased number of 
measurements are used with the intention of better approximating the trunk segment for female subjects. Note that Yeadon's model was only validated using direct whole-body measures. ${ }^{9}$ The primary hypothesis is that trunk BSIPs predicted by a geometric solid model with an increased number of trunk measurements would improve the accuracy of female trunk masses predicted using geometric modeling compared with those determined using DXA. In particular, using the method of Bland and Altman analysis, the predicted BSIP would be within the $95 \%$ confidence interval. The secondary aim of this study was to determine whether a reduced number of limb measurements can be taken while preserving the accuracy of BSIPs for the whole body and model segments using a reduced number of geometric solids.

\section{Methods}

Twenty-eight white female athletes volunteered for the study (mean age of 24 years, SD 4.7). The mean body mass of the group was $64.4 \mathrm{~kg}$ (SD 7.5), mean height was $1.65 \mathrm{~m}$ (SD 5.6), and mean body mass index (BMI) was $23.8 \mathrm{~kg} / \mathrm{m}^{2}$ (SD 3.1). All participants gave written informed consent and the procedures were approved by the Ethics Committee for Research Procedures at Aberystwyth University. Participants wore tight-fitting bathing suits during all measurements.

The model was validated by Bland-Altman ${ }^{14}$ analyses comparing: model-predicted whole-body volume with that determined by underwater weighing; model-predicted whole-body center of mass with that determined by a reaction board; model-predicted whole-body mass with that obtained via direct measurement using scales; and model-predicted segment masses with those estimated from whole-body DXA scans.

The geometric model comprised 15 segments which were in turn divided into a number of geometric solids. The full model comprised 56 geometric solids, the reduced model comprised 31 geometric solids, and the basic model comprised 16 geometric solids (Table 1). To determine the inertial properties of the geometric solids, a total of 118 anthropometric measures were made on each participant. For the full model, the trunk segment was modeled as a series of 10 stacked stadium solids. ${ }^{9}$ The stadium solid requires the measurement of the width and the perimeter of the torso in order to define the stadium outline of the stadium solid base. ${ }^{9}$ The anthropometric measurements were taken at points on the trunk chosen to best map the shape of the female trunk; the transverse measures (perimeter and width) were taken at the clavicular notch, oxter (armpit), above bust, across nipples, under bust, seventh rib, waist, navel, iliac crest, ASIS, and the greater trochanter. The vertical (with the participant in a standing position) distances along the torso between the level of these anatomical landmarks were measured. For the reduced model the trunk was modeled as a series of 5 stacked stadium solids, extending between the clavicular notch, above bust, under bust, waist, iliac crest, and the greater trochanter. For the basic model, the trunk was modeled as a series of 2 stacked stadium solids extending between the clavicular notch, the waist, and the greater trochanter. The $8 \mathrm{limb}$ segments (upper arm, forearm, thigh, and shank) were divided into truncated cones of equal length along the segment: the full model had 4 truncated cones per segment, the reduced model had 2, and the basic model had 1 truncated cone (Figure 1). For example, for the full model for the shank, the circumference was measured at the ends of the segment corresponding to the approximate joint centers (the lateral femoral condyle and the lateral malleolus). The distance in between these 2 landmarks was measured and the distance was
Table 1 The Geometric Solids Used to Model the Individual Segments for the Full, Reduced, and Basic Models

\begin{tabular}{llll}
\hline Segment & Full model & Reduced model & Basic model \\
\hline Upper arms & 4 truncated cones & 2 truncated cones & 1 truncated cone \\
Forearms & 4 truncated cones & 2 truncated cones & 1 truncated cone \\
Hands & 3 stadium solids & 2 stadium solids & 1 stadium solid \\
Thighs & 4 truncated cones & 2 truncated cones & 1 truncated cone \\
Shanks & 4 truncated cones & 2 truncated cones & 1 truncated cone \\
Feet & 3 stadium solids & 2 stadium solids & 1 stadium solid \\
Trunk & 10 stadium solids & 5 stadium solids & 2 stadium solids \\
Head & 1 ellipsoid & 1 ellipsoid & 1 ellipsoid \\
Neck & 1 truncated cone & 1 truncated cone & 1 truncated cone \\
\hline
\end{tabular}

divided by 3 so that the distance a quarter, a half, and three quarters of the way along the segment could be identified (giving 4 truncated cones). Circumferences were then taken at these points. The reduced model included the circumferences at the ends and in the middle of the segment ( 2 truncated cones), and the basic model only included the circumferences at each end of the segment (one truncated cone). The hands and feet were modeled as a series of stadium solids of equal lengths: 3 for the full model, 2 for the reduced model, and 1 for the basic model (Figure 1). The model uses the same geometric shapes for each body segment as the model of Yeadon, ${ }^{9}$ yet differs in that the segments are modeled with more solids in series for each body segment; the reduced model is most similar to that of Yeadon ${ }^{9}$ except for a more detailed model of the trunk. More specific details of model code are available from the first author, on request.

All measurements on the subjects were made once by an appropriately trained investigator. For all segments the landmark positions were marked using a nonpermanent fine line marker or tailor's chalk. Measurements were taken to the nearest $0.5 \mathrm{~mm}$ using an anthropometric measuring tape and calipers, with participants standing in the anatomical position.

In order to determine segment masses, segment volumes (determined by the geometric shape used) were multiplied by appropriate segmental densities from Clauser et al ${ }^{15}$ data. The center of mass location along the major axes of each segment was computed. The computed segment volumes, masses, and center of masses were summed to predict whole-body volume and wholebody mass, and used to determine whole-body center of mass. All computations were made using custom written MATLAB software (The Mathworks, Inc, Natick, MA).

Criterion whole-body mass was determined using a calibrated set of scales and recorded to the nearest $0.01 \mathrm{~kg}$. Whole-body center of mass, along the long axis, was determined using the reaction board technique. ${ }^{16}$ Subjects lay supine with palms of the hands flat against the proximal side of the thighs.

Whole-body volume was measured using the hydrostatic weighing technique. Participants were familiarized with the water tank and the procedure and were allowed to practice the technique until they felt comfortable. Underwater weight was measured when the subjects were fully submerged, had breathed out, were motionless, and the load cell display stabilized on a value. Three measurements were taken with the maximum value taken as the underwater weight. Adjustments were made for water temperature. Residual lung volume was estimated using height and age, and intestinal 
Table 2 The Whole-Body Inertial Properties (Means \pm SD), Measured by Underwater Weighing (Volume), Scales (Mass), and Reaction Board (Center of Mass Location), and Those Estimated by the 3 Geometric Solid Models*

\begin{tabular}{|c|c|c|c|c|}
\hline & Criterion measure & Full model & Reduced model & Basic model \\
\hline Whole-body volume (L) & $63.4 \pm 7.9$ & $62.3 \pm 8.0$ & $63.9 \pm 7.7$ & $62.4 \pm 7.3$ \\
\hline Whole-body mass (kg) & $66.0 \pm 7.6$ & $67.2 \pm 8.5$ & $66.2 \pm 8.2$ & $62.5 \pm 7.5$ \\
\hline Whole-body center of mass location (m) & $0.91 \pm 0.04$ & $0.94 \pm 0.04$ & $0.91 \pm 0.04$ & $0.91 \pm 0.04$ \\
\hline
\end{tabular}

Note. Full trunk model is used for each of the 3 geometric solid models.

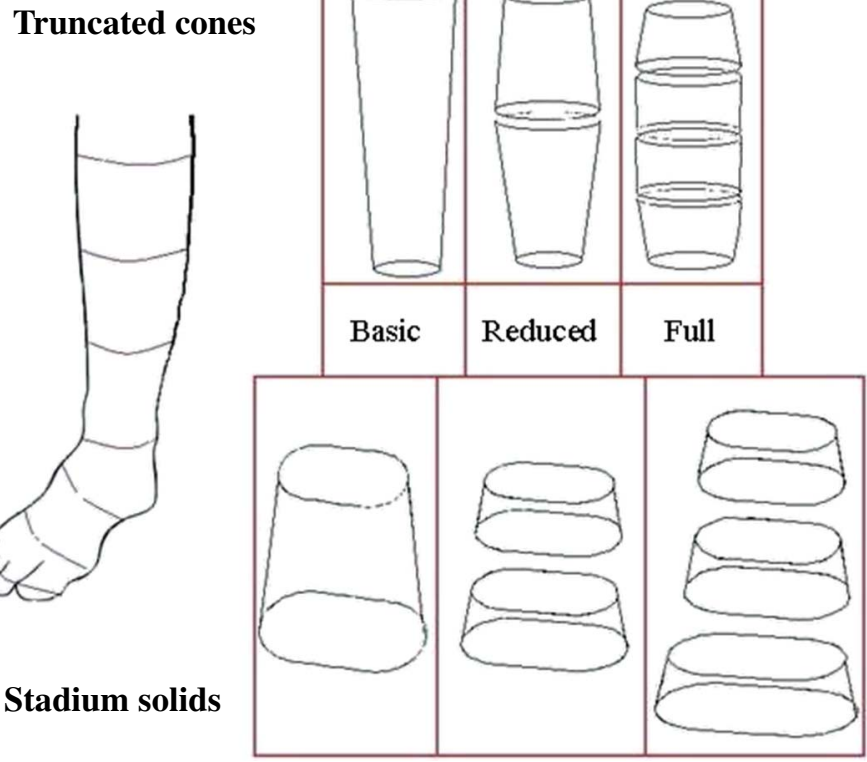

Figure 1 - The composition of the basic, reduced, and full models for the limbs: an example showing the shank and foot segments, which were composed of truncated cones and stadium solids, respectively.

gases were assumed to be $100 \mathrm{ml} .{ }^{17}$ Whole-body volume and density were calculated using the equations from Siri. ${ }^{18}$

Participants underwent a whole-body DXA scan (Hologic Discovery; Hologic UK Ltd, Sussex, UK). The system had a scan pixel resolution of $1.2 \mathrm{~mm} \times 1.8 \mathrm{~mm}$, and a mass resolution of $0.01 \mathrm{~g} / \mathrm{mm}^{2}$. The scan was performed with the subjects lying supine on the table with forearms pronated so that the palms of their hands were facing the lateral side of their thighs. The output from each scan was the distribution of mass of the whole body. Each scan was digitized in order to locate bony landmarks and section the body segments to correspond with the anthropometric data collected, giving the mass of each segment. One trained operator performed all of the segmentation of the scans.

Whole-body volume, whole-body mass, and whole-body center of mass were determined for each of the geometric solid models. These measures were compared with whole-body mass measured with scales, whole-body center of mass location measured via a reaction board, and whole-body volume measured via underwater weighing. Whole-body density was computed and compared with the whole-body density calculated from the hydrostatic weighing procedure. Segmental volumes and masses were compared with those estimated from DXA scans.

Normality of the data was assessed using an Anderson Darling test of normality. All variables were normally distributed. The segment mass data for each method was normalized by dividing by the DXA-derived whole-body mass. Percentage root mean square errors (RMSE) are reported for the normalized estimates from each model compared with the normalized DXA data, and for direct measures of whole-body mass, whole-body volume, and center of mass location compared with estimates from each model. The method of Bland and Altman ${ }^{14}$ was used in assessing each comparison. Where applicable, $95 \%$ confidence intervals for the percentage deviation between methods are reported. All statistical analysis was performed in Minitab v17 (Minitab@ Statistical Software, State College, PA, USA).

\section{Results}

The RMSE of the trunk mass predicted by the full geometric model compared with that reported on the DXA scan was $3.4 \%$ (Tables 2 and 3), which is similar to the possible operator error due to

Table 3 Segment Data From the Whole-Body DXA Scans and \% RMSEs for Each Segment of the Geometric Model Compared With DXA Data

\begin{tabular}{|c|c|c|c|c|c|}
\hline \multirow[b]{2}{*}{ Segment } & \multicolumn{2}{|c|}{ DXA segment data (\% whole-body mass) } & \multicolumn{3}{|c|}{ Geometric model RMSE (\% segment mass) } \\
\hline & Mean & SD & Full & Reduced & Basic \\
\hline Upper arm & 3.1 & 0.35 & 10.2 & 12.8 & 23.1 \\
\hline Forearm & 1.3 & 0.03 & 5.9 & 6.8 & 12.1 \\
\hline Hand & 0.5 & 0.11 & 13.7 & 13.8 & 36.1 \\
\hline Thigh & 9.1 & 0.75 & 11.9 & 11.1 & 9.5 \\
\hline Shank & 4.9 & 0.43 & 11.6 & 17.1 & 9.5 \\
\hline Foot & 1.2 & 0.15 & 5.0 & 7.3 & 21.6 \\
\hline Trunk & 54.7 & 2.19 & 3.4 & 7.1 & 16.6 \\
\hline Head and neck & 7.3 & 0.92 & 7.1 & 7.1 & 7.1 \\
\hline
\end{tabular}

Abbreviations: DXA, dual x-ray absorptiometry; RMSE, root mean square error.

Note. Limb segments are means of the left and right sides; the head/neck segment was estimated in the same way for all 3 geometric models. 


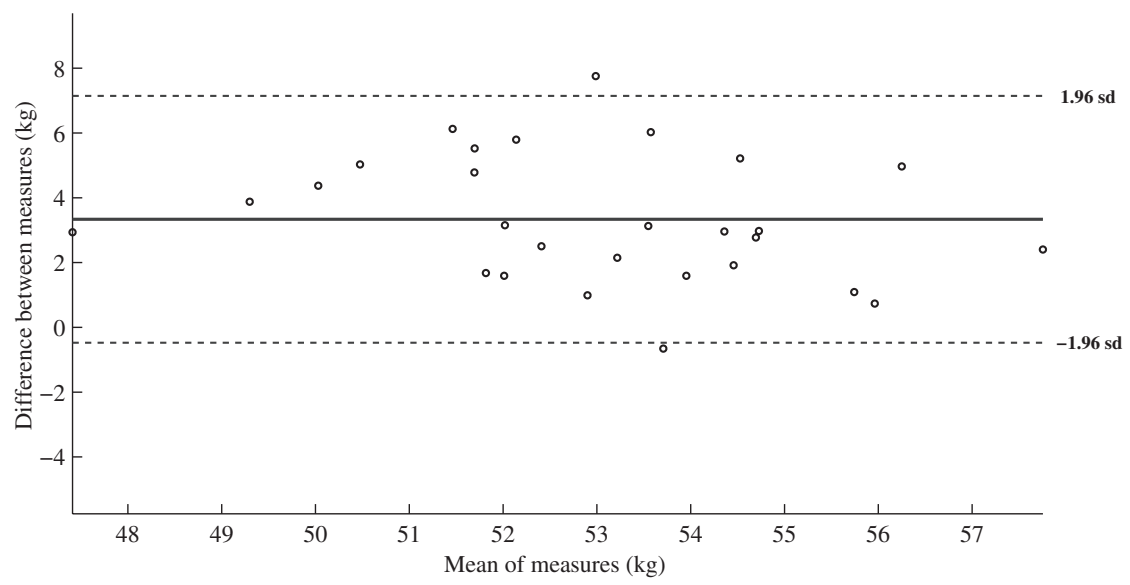

Figure 2 - Agreement of normalized dual x-ray absorptiometry derived trunk segment mass with normalized geometric full model trunk predictions using the method of Bland and Altman. ${ }^{14}$

participant positioning and scan analysis. ${ }^{19}$ The Bland-Altman ${ }^{14}$ plot for the full trunk model shows that all of the data points lie between or very close to the $95 \%$ limits of agreement, and that there is no residual trend across the range of predicted trunk masses as a percentage of whole-body mass (Figure 2). The 95\% confidence interval for the difference in trunk mass as a percentage of wholebody mass between the full geometric model and the DXA scan was $1.97 \%, 0.27 \%$, indicating that the geometric model slightly underestimated the trunk mass in comparison to the DXA scan predicted mass. The RMSE of the trunk mass for the reduced and basic models were $7.1 \%$ and $16.6 \%$, respectively.

Given the large errors associated with the trunk mass for the reduced and basic trunk model, the whole-body figures are reported for the full, reduced, and basic limb segments but incorporating the full trunk model for all 3 models, so that the effect of reducing the limb measurements can be explored. The RMSE for whole-body volume was less than $2.5 \%$ of whole-body volume for all 3 models, and $1.9 \%(1.5 \mathrm{~L})$ for the full geometric model (Table 4$)$. The $95 \%$ confidence interval for the mean difference between the tank measured volume and the full geometric model was $0.9 \%, 2.4 \%$ (\% of tank predicted volume) indicating the geometric model slightly underestimated the whole-body volume predicted by the underwater weighing method. The RMSE whole-body mass was

Table 4 The Errors in Whole-Body Volume, Mass, and Center of Mass Location, Expressed as the Percentage Root Mean Square Error (\% RMSE) for the 3 Geometric Solid Models Compared With Direct Measures From Underwater Weighing, Scales, and a Reaction Board, Respectively*

\begin{tabular}{lccr}
\hline & \multicolumn{3}{c}{ \%RMSE in model predictions } \\
\cline { 2 - 4 } & $\begin{array}{c}\text { Full } \\
\text { model }\end{array}$ & $\begin{array}{c}\text { Reduced } \\
\text { model }\end{array}$ & $\begin{array}{r}\text { Basic } \\
\text { model }\end{array}$ \\
\hline Whole-body volume & 1.9 & 2.5 & 1.9 \\
Whole-body mass & 3.1 & 5.1 & 3.4 \\
Whole-body center of & 3.2 & 2.1 & 2.7 \\
mass location & & & \\
Whole-body density & 1.2 & 1.3 & 1.3 \\
\hline
\end{tabular}

Note. Full trunk model is used for each of the 3 geometric solid models.
$3.1 \%$ for the full model, and was larger for the reduced and basic models (Table 4). The RMSE for center of mass location was 3.2\% or less for all models (Table 4).

The mean difference between geometric solid model and DXA measures as a percentage of whole-body mass was less than $0.5 \%$ for all segments (Figures 3 and 4). The segments with the best agreement in terms of the Bland-Altman plots were the thigh, forearm, and hand segments, with, at most, 1 data point lying away from the $95 \%$ confidence limits, and the agreement was similar for both the full and reduced models. The full and reduced models were also similar in terms of the RMSE for these (thigh, forearm, and hand) segments (Table 3). The RMSE as a percentage of segment mass was highest for the thigh at $11.9 \%$ of segment mass; this is equivalent to just $0.7 \mathrm{~kg}$ for the mean whole-body mass in the present participant pool.

The RMSE as a percentage of segment mass increased for both the upper arm and the shank when moving from the full model to the reduced model. The RMSE for the full model for these segments was comparable to other segments (11\% of segment mass, which is equivalent to $0.3 \mathrm{~kg}$ for the mean whole-body mass in the present participant pool). The Bland-Altman plots for the full model for the upper arm and shank segments showed no residual trend; mean differences between the geometric model and DXA predictions were close to zero, and there was good agreement with only 2 to 3 points outside the $95 \%$ limits of agreement. For the upper arm and shank segments, the width of the $95 \%$ limits of agreement were less than $1.5 \%$ of whole-body mass.

\section{Discussion}

The purpose of this work was to validate a geometric solid-based body segment inertial parameter model, based on that of Yeadon, ${ }^{9}$ by comparing whole-body model predictions with direct measurements, and by comparison of segmental predictions with DXA-derived predictions. The present study is important as geometric models have the potential to predict subject-specific inertial parameters at a low cost within a reasonably short data collection time using widely-available equipment. The primary hypothesis was that trunk BSIPs predicted by a geometric solid model with an increased number of trunk measurements would improve the accuracy of female trunk masses predicted using geometric modeling compared with those determined using 

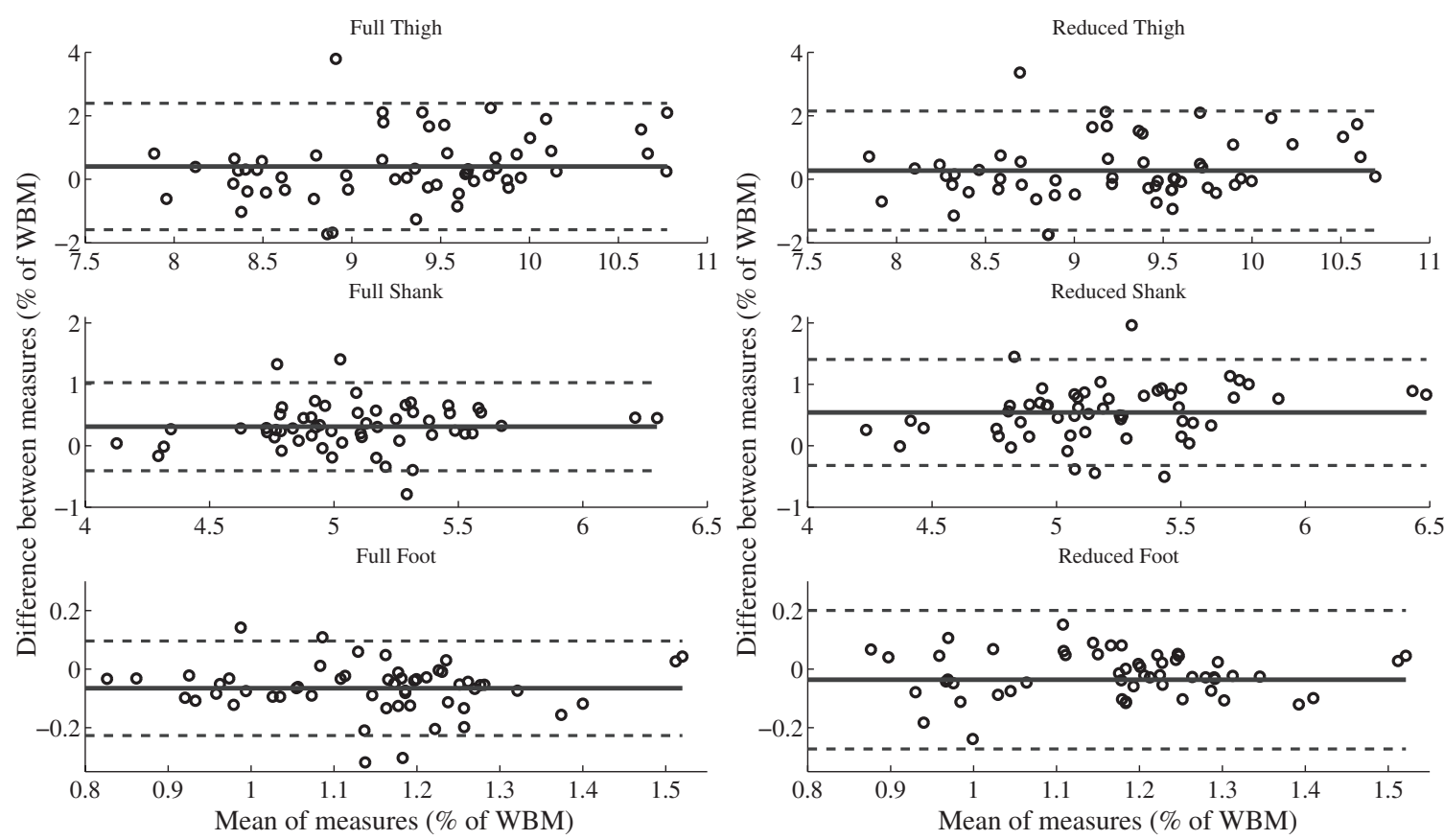

Figure 3 - Agreement of normalized dual x-ray absorptiometry derived segment masses with normalized full and reduced model predictions for the thigh, shank, and foot using the method of Bland and Altman. ${ }^{14}$
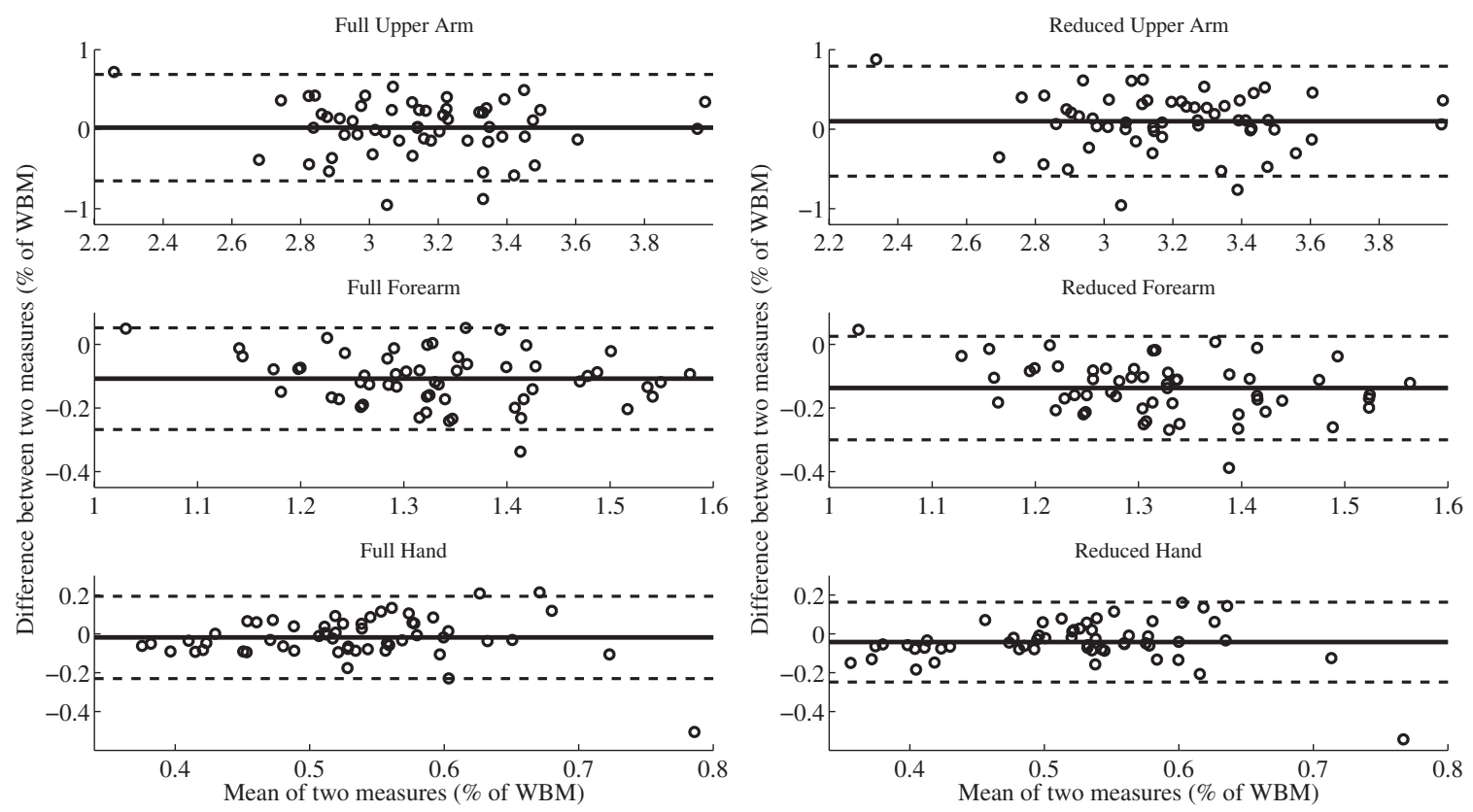

Figure 4 - Agreement of normalized dual x-ray absorptiometry derived segment masses with normalized geometric full and reduced model predictions for the upper arm, forearm, and hand using the method of Bland and Altman. ${ }^{14}$

DXA, and it was shown that the reduced and basic trunk models more than doubled and more than quadrupled the RMSE for trunk mass, respectively.

The RMSE for whole-body volume was just $2.3 \%$, which compares favorably with the accuracy of previous models that were validated for far fewer participants. ${ }^{7-9}$ For example, the models of $\mathrm{Hatze}^{7}$ and Yeadon ${ }^{9}$ were both validated using only whole-body measures for 1 female subject. The RMSE in whole-body mass of
$3.3 \%$ is slightly higher than that reported by Yeadon ${ }^{9}(2.3 \%)$, but the present model has been validated here using 28 subjects rather than the 3 used by Yeadon. ${ }^{9}$ Hatze $^{7}$ claimed a mean error in whole-body mass of $0.26 \%$, however this model was only validated for 3 adults and involves taking a considerable number (242) of anthropometric measurements. Neither of these models reported validation of individual segment masses since appropriate techniques were not available at the time. Notably, in the present study, the RMSE 
between the scales and the geometric model predicted whole-body mass was similar to the RMSE between the scales and the DXA estimated whole-body mass of $\sim 2.5 \%$. The greater RMSE for whole-body mass than for whole-body volume may be due to the segmental densities used in the geometric model ${ }^{20}$; the data used here ${ }^{15}$ was based on male cadaver data and unfortunately most available segmental density data sets have been derived from males.

The present study was in part motivated by pilot work showing an RMSE for whole-body volume of $15 \%$ using the models of Hanavan $^{21}$ and Yeadon ${ }^{9}$ for a sample of 10 females. To our knowledge the model presented here is the first geometric model aimed specifically at females, and has produced the lowest reported RMSE in predicted trunk segment mass using a uniform density model (3.4\% of segment mass). Using a female-specific nonuniform density function could possibly reduce errors further, but the use of nonuniform density data has been shown to only affect errors in predicting lower trunk mass. ${ }^{20}$

The RMSE for the thigh segment for the full and reduced model was $11.9 \%$ and $11.1 \%$, respectively. This is lower than the RMSE of $18.3 \%$ reported by Durkin et $\mathrm{al}^{22}$ for a previous geometric thigh model. The RMSE for the shank segment mass for the present model was $11.6 \%$ for the full model. A previous geometric shank model validated by comparison with DXA data reported mean errors of $2.83 \%$ for segment mass for females aged 19-30 years. ${ }^{10}$ However, that paper reported mean error, as opposed to RMSE, and error reported using the former measure is likely to be smaller. Additionally, the present study validation involved a larger participant pool.

In general, the RMSE percentage errors for all segment masses represented a mass less than $0.3 \mathrm{~kg}$. The influence of errors in the estimation of BSIP depends on the purpose of the analysis and the task being examined. For example, a simulation model may not require subject-specific BSIPs, in which case BSIPs within a feasible range may be appropriate; if the model is subject-specific the appropriateness of the BSIP can be indicated by comparison of model performance with subject performance. ${ }^{9}$ In certain tasks (for example, a dumbbell curl), the inertial properties of the dumbbell dominate those of the hand and forearm, in which case accurate dumbbell inertial properties are more important than hand and forearm. ${ }^{23,24}$ However, for some movements, errors in the BSIPs can have an influence on the computed resultant joint moments. ${ }^{25}$ The present model represents an improvement in previously reported geometric models for female subjects given that, with the additional trunk and limb measures described here, the RMSE for segment mass can be reduced. However, given the paucity of female-specific segment density data, and yet the importance of accurate BSIP estimates reported by Reinbolt et al, ${ }^{25}$ it is clear that the refinement of anthropometric models continues to be an important area of work.

A second important aim of this work was to determine the effect of reducing the number of truncated cones forming each of the limb segments, and the number of stadium solids forming the hand and foot segments. This has the practical effect of reducing the number of circumference measurements taken for each limb, which significantly reduces data collection time. The RMSE for the thigh, forearm, upper arm, foot, and hand were low for both the full and reduced models, and the Bland-Altman ${ }^{14}$ plots show good agreement for both models for these segments. Researchers seeking to minimize time spent with participants may prefer to take only end and middle of segment circumferences for these segments.

The RMSE for the shank increased considerably when moving from the full to the reduced model. It is somewhat paradoxical that the RMSE then decreased considerably (and below that of the full model) when moving to the basic model for the shank. A previous geometric model of the shank has previously been shown to require 3 geometric solids to produce low mean segment mass errors. ${ }^{10}$ Taken together, these studies suggest that the change in diameter along the shank segment is sufficiently unpredictable that several measurements are required to accurately predict inertial parameters using a geometric model. Given the alteration in RMSE from the full to the reduced model, and the previously reported findings, it is likely the lower RMSE for the basic model represents a canceling out of overestimations and underestimations along the segment. This is hard to show conclusively since the DXA scan represents a projection of a 3-dimensional volume onto a 2-dimensional plane, which makes it difficult to draw conclusions about the mass distribution. However, it is possible that the basic shank model, while benefiting from a lower \%RMSE for mass, may not reflect mass distribution well, and may therefore not accurately estimate other parameters that cannot be validated using DXA, such as center of mass location and the moments of inertia.

Summing the DXA-derived segmental masses resulted in a mean whole-body mass of $101.5 \%$ of the whole-body mass reported by the DXA software. This issue has previously been reported by Wicke and Dumas ${ }^{26}$ and Chambers et al, ${ }^{27}$ who found that DXA-derived segment masses and lengths do not sum to whole-body mass and height determined using traditional scales and stadiometers, respectively. It is likely that this is due to pixels at segment boundaries being included in both bordering segments. At the segmental level, a likely source of discrepancies between DXA and geometric model derived data is due to differences in soft tissue distribution when lying down compared with standing upright. In many sporting and daily activity situations, the soft tissue distribution is more likely to reflect that in the upright position. Furthermore, there is an inherent difference between the 2 techniques in that the anthropometric measurements predict volume, from which mass is calculated via an assumed density. Conversely, the DXA method measures absorption and, hence, area, from which mass is calculated using an assumed density. In fact, some authors argue that there has been insufficient validation of DXA measurements, ${ }^{6}$ and, unfortunately, while previous work using DXA data has benefited from access to raw pixel data, eg, Durkin et al, ${ }^{4}$ DXA manufacturers are increasingly reluctant to allow such access generally. For these reasons, DXA-derived measurements cannot necessarily be seen as the gold standard, but instead simply as a reasonable comparator.

A range of methods have been used to estimate body segment inertial parameters for biomechanical analyses. Cadaver-based regression equations have been shown to produce large errors particularly for young adults and females and do not fully account for morphological differences. ${ }^{5,22}$ Later regression equations based on gamma mass scanning did not consistently produce more accurate results and do not reflect individual morphology differences within population groups. ${ }^{5}$ Direct measurements using technologies such as DXA and magnetic resonance imaging have been shown to produce accurate whole-body results, ${ }^{4,28}$ but such technologies have limited availability due to high costs and the training required to use them. Geometric models offer the opportunity for estimating subject-specific BSIPs at low cost with reasonable accuracy, and the present work represents the first large-scale validation of such a model for young females.

In conclusion, a geometric body segment inertial parameter model based on that of Yeadon, ${ }^{9}$ but including additional trunk and limb measurements specifically designed for female participants, 
was validated for 28 young female college athletes. The validation compared predicted values with direct and indirect measurements for whole-body volume, mass, and center of mass predictions, and DXA for segmental mass predictions. Percent RMSE for wholebody and segment measures were comparable with or lower than previous geometric models. Researchers seeking to reduce time spent taking anthropometric measurements may wish to reduce the number of truncated cones used to model some segments; however, care should be taken when reducing the number of solids as some segments displayed greater sensitivity than others to reducing the number of solids used.

\section{References}

1. Ganley KJ, Powers CM. Determination of lower extremity anthropometric parameters using dual energy X-ray absorptiometry: the influence on net joint moments during gait. Clin Biomech. 2004;19(1):50-56. PubMed doi:10.1016/j.clinbiomech.2003.08.002

2. Pearsall DJ, Costigan PA. The effect of segment parameter error on gait analysis results. Gait Posture. 1999;9(3):173-183. PubMed doi:10.1016/S0966-6362(99)00011-9

3. Bellemare F, Jeanneret A, Couture J. Sex differences in thoracic dimensions and configuration. Am J Respir Crit Care Med. 2003;168(3):305-312. PubMed doi:10.1164/rccm.200208-876OC

4. Durkin JL, Dowling JJ, Andrews DM. The measurement of body segment inertial parameters using dual energy X-ray absorptiometry. J Biomech. 2002;35(12):1575-1580. PubMed doi:10.1016/S00219290(02)00227-0

5. Durkin JL, Dowling JJ. Analysis of body segment parameter differences between four human populations and the estimation errors of four popular mathematical models. $J$ Biomech Eng. Aug 2003;125(4):515-522. PubMed doi:10.1115/1.1590359

6. Scafoglieri A, Provyn S, Wallace J, et al. Whole body composition by Hologic QDR 4500/A DXA: system reliability versus user accuracy and precision. In: Ivanov PO, ed. Applications and Experiences of Quality Control. Rijeka, Croatia: InTech; 2011:45-62.

7. Hatze H. A mathematical model for the computational determination of parameter values of anthropomorphic segments. J Biomech. 1980;13(10):833-843. PubMed doi:10.1016/0021-9290(80)90171-2

8. Jensen RK. Estimation of the biomechanical properties of three body types using a photogrammetric method. J Biomech. 1978;11 (8-9):349-358. PubMed doi:10.1016/0021-9290(78)90069-6

9. Yeadon MR. The simulation of aerial movement-II. A mathematical inertia model of the human body. J Biomech. 1990;23(1):67-74. PubMed doi:10.1016/0021-9290(90)90370-I

10. Durkin J, Dowling J. Body segment parameter estimation of the human lower leg using an elliptical model with validation from DEXA. Ann Biomed Eng. 2006;34(9):1483-1493. PubMed doi:10. 1007/s10439-006-9088-6

11. Wicke J, Dumas GA, Costigan PA. A comparison between a new model and current models for estimating trunk segment inertial parameters. J Biomech. 2009;42(1):55-60. PubMed doi:10.1016/ j.jbiomech.2008.10.003
12. Wei C, Jensen RK. The application of segment axial density profiles to a human body inertia model. J Biomech. 1995;28(1):103-108. PubMed doi:10.1016/0021-9290(95)80012-3

13. Cornelis J, VanGheluwe B, Nyssen M, Van DenBerghe F. A Photographic Method For The Tridimensional Reconstruction Of The Human Thorax. Paper presented at: NATO Symposium on Applications of Human Biostereometrics; July 29, 1980; Paris, France.

14. Bland JM, Altman DG. Measuring agreement in method comparison studies. Stat Methods Med Res. 1999;8(2):135-160. PubMed doi:10. 1177/096228029900800204

15. Clauser CE, McConville JT, Young JW. Weight, Volume and Center of Mass of Segments of the Human Body. AMRL-TR-69-70. Dayton, OH: Aerospace Medical Research Laboratory, Wright-Patterson Air Force Base; 1969.

16. Winter DA. Biomechanics and Motor Control of Human Movement. 4th ed. Chichester, UK: Wiley; 2009:82-106.

17. Miller WC, Swensen T, Wallace JP. Derivation of prediction equations for RV in overweight men and women. Med Sci Sports Exerc. 1998; 30(2):322-327. PubMed doi:10.1097/00005768-199802000-00023

18. Siri W. Body composition from fluid spaces and density. In: Brozek J, Henschel A, eds. Techniques for Measuring Body Composition. Washington, DC: National Academy of Science; 1961:223-244.

19. Forrest SM. The validation of biomechanical methods for ageing and sex: force steadiness and body segment inertial parameters. $[\mathrm{PhD}$ Thesis]. Aberystwyth, United Kingdom: Aberystwyth University; 2012.

20. Wicke J, Dumas GA. Influence of the volume and density functions within geometric models for estimating trunk inertial parameters. $J$ Appl Biomech. 2010;26(1):26-31. PubMed doi:10.1123/jab.26.1.26.

21. Hanavan EP Jr. A Mathematical Model of the Human Body. Amrl-Tr64-102. AMRL TR. 1964:1-149. PubMed

22. Durkin JL, Dowling JJ, Scholtes L. Using mass distribution information to model the human thigh for body segment parameter estimation. J Biomech Eng. 2005;127(3):455-464. PubMed doi:10. $1115 / 1.1894367$

23. Challis JH. Accuracy of human limb moment of inertia estimations and their influence on resultant joint moments. J Appl Biomech. 1996;12:517-530. doi:10.1123/jab.12.4.517

24. Challis JH, Kerwin DG. Quantification of the uncertainties in resultant joint moments computed in a dynamic activity. J Sports Sci. 1996;14(3):219-231. PubMed doi:10.1080/02640419608727706

25. Reinbolt JA, Haftka RT, Chmielewski TL, Fregly BJ. Are patientspecific joint and inertial parameters necessary for accurate inverse dynamics analyses of gait? IEEE Trans Bio-med Eng. 2007;54(5):782-793. PubMed doi:10.1109/TBME.2006.889187

26. Wicke J, Dumas GA. Estimating segment inertial parameters using fan-beam DXA. J Appl Biomech. 2008;24(2):180-184. doi:10.1123/ jab.24.2.180

27. Chambers AJ, Sukits AL, McCrory JL, Cham R. The effect of obesity and gender on body segment parameters in older adults. Clin Biomech. 2010;25(2):131-136. doi:10.1016/j.clinbiomech.2009.10.015

28. Mungiole M, Martin PE. Estimating segment inertial properties: comparison of magnetic resonance imaging with existing methods. J Biomech. 1990;23(10):1039-1046. PubMed doi:10.1016/00219290(90)90319-X 